\title{
Age and gender normative data for lift capacity
}

\author{
Leonard N. Matheson ${ }^{\mathrm{a}, \mathrm{b}, *}{ }$, Joe Verna ${ }^{\mathrm{a}, \mathrm{c}}$, Thomas E. Dreisinger ${ }^{\mathrm{a}}$, Scott Leggett ${ }^{\mathrm{a}}$ and John Mayer ${ }^{\mathrm{a}, \mathrm{d}}$ \\ ${ }^{a}$ Vert Mooney Research Foundation, San Diego, CA, USA \\ ${ }^{\mathrm{b}}$ EpicRehab, LLC, St. Louis, MO, USA \\ 'Spine and Sport, San Diego, CA, USA \\ ${ }^{\mathrm{d}}$ University of South Florida, Tampa, FL, USA
}

Received 13 December 2012

Accepted 14 March 2013

\begin{abstract}
.
BACKGROUND: Functional Capacity Evaluations (FCEs) are routinely used in physical medicine to ascertain an individual's work ability; with lift capacity being an important aspect of many evaluations. Despite the widespread use of lift capacity tests, there are few studies that provide age and gender normative data.

OBJECTIVE: To evaluate the safety, reliability, and validity of the EPIC Lift Capacity test, and to examine the effects of age and gender on lift capacity.

METHODS: This study used a test-retest design with 4,443 adult participants in good general health. Test-retest of participants' lift capacity was undertaken to examine safety and reliability. Age and gender and the self-reported physical demands of each employed participant's usual and customary job were collected to examine validity.

RESULTS: Safety and reliability were demonstrated for both measures of lift capacity for each of the six sub-tests in the EPIC Lift Capacity test battery. Inter-subtest differences and expected age and gender differences were found across all subtests.

CONCLUSIONS: The EPIC Lift Capacity test is a safe and reliable test of lift capacity. Normative data are presented that allow comparison within age and gender categories.
\end{abstract}

Keywords: Functional Capacity Evaluation, test safety, test reliability, norms, normal values

\section{Introduction}

Functional Capacity Evaluations (FCEs) are routinely used to measure the ability of an individual to perform work-related tasks, with lift capacity being an important aspect of many evaluations. Despite the widespread use of lift capacity tests, there are few studies that provide age and gender normative data.

Research on the psychometric properties of FCEs has resulted in more than 70 studies, which have been summarized by Innes [1-3]. In an extensive review of

\footnotetext{
*Corresponding author: Dr. Leonard Matheson, EpicRehab, 188 Woodland Place Court, St. Charles, MO 63303, USA. Tel.: +1 636 346 8869; Fax: +1 636898 0954; E-mail: Len@epicrehab.com.
}

the literature, Innes [4] updated these studies and a clinician's guide [5], including interval studies that appeared in peer-reviewed publications between January 1998 and March 2006 on the reliability and validity of individual tests used in functional capacity evaluation and standardized FCE test batteries. Studies of reliability are more frequent than in prior years, generally demonstrating "moderate to excellent levels of reliability, particularly manual/material handling components." Validation studies continue to be rare, with weak relationships between test performance and return to work in some studies [6,7] and an absence of relationship to maintenance at work in other studies $[8$, 9]. None of these studies presented normative data.

Normative data are used throughout healthcare and 
rehabilitation with measures of grip strength [10-12] and fitness [13-16]. Normative data for these instruments are analyzed with reference to appropriate covariates that are likely to affect response to the test, which are used to stratify the data in a meaningful way. For example, an evaluee's grip strength is compared with age-referenced and gender-referenced normative data to provide an estimate of the evaluee's likely functional loss as a result of injury.

Age is a variable that is implicated in strength testing $[17,18]$ and must be considered whenever lifespan normative data are presented. As a category of human performance that depends on many body systems that change with age, it is reasonable to expect that substantial age changes will be found with lift capacity, demonstrated as differences in cross-sectional studies. In order to perform valid normative comparisons, the extent of age differences in lift capacity must be known. Matheson [19] provided normative data for the West Standard Evaluation and the EPIC Lift Capacity Test (ELC) [20]. Mayer et al. [21] provided normative data for the Progressive Isoinertial Lifting Evaluation. However, only the more recent Matheson study stratified performance in terms of both age and gender. The current paper extends that study with a larger sample. The purpose of this study is to evaluate the safety, reliability, and validity of the ELC, and to examine the effects of age and gender on lift capacity in order to provide appropriately stratified normative lift capacity data.

\section{Background}

Lifting, "the act of manually grasping and raising an object of definable size" [22], is a component of many functional capacity evaluations because judgment about safety in jobs that require lifting requires a comparison of the worker's abilities and the job's demands [23]. Likewise, lift capacity testing is a component of many different types of occupational rehabilitation treatment programs [24-28]. To provide utility, such tests must meet widely agreed-upon criteria for safety, reliability, validity, and practicality [22, 29]. In order of importance, these tests must have been demonstrated to be safe with people who have already experienced an injury [30-32], reliable in these applications [33-35], and must provide valid answers for the issues the test is intended to measure, including the availability of normative data [36]. Practicality is based on the resources that must be expended to meet these criteria. The utility of a measure of lift capacity in rehabilitation is based on the degree to which it meets these criteria and the data can be used to:

1. Gauge treatment effect by comparing the baseline level of performance with performance as treatment progresses and at the conclusion of the treatment;

2. Set treatment goals that are reasonable within the context of normal values for patients of the same age and gender;

3. Estimate post-injury loss of function and work disability by comparing the patient's performance to expected values;

4. Make recommendations for work assignment by comparing the person's lift capacity to the job's demands.

While the first and last of these purposes can be achieved without reference to normative values, the second and third purposes are only possible with agebased and gender-based normative data.

\subsection{Equipment design}

The Epic Lift Capacity (ELC) equipment are standardized and described in an examiner's manual [3739], where the formal test procedures are also presented. The test requires a standardized steel-reinforced lifting crate with offset handles and a set of masked weights, a heart rate monitor, and sturdy shelves. Color-coded masked weight containers weighing $2.3 \mathrm{~kg}$ (5 lbs) to $6.8 \mathrm{~kg}$ (15 lbs) of exactly the same exterior dimensions are used. The empty weight of the ELC crate is $4.5 \mathrm{~kg}$ (10 lbs).

\subsection{Evaluation process}

The ELC is conducted on a blind basis beginning with an empty crate at $4.5 \mathrm{~kg}$ (10 lbs) and increasing in $4.5 \mathrm{~kg}$ increments (loaded by the evaluator) for all evaluees. The evaluee is not told the beginning weight or the weight increments. The combinations of starting height and range (achieved by adjusting the shelves) are standardized into three categories, based on the participant's height over three vertical ranges; waist to shoulder, floor to waist, and floor to shoulder. The frequency of the ELC is one lift per cycle or four lifts per cycle, depending on the subtest. To minimize the effect of accumulated work, the starting loads for the later tests in the battery are proportionally based on the maximum acceptable weight attained in the earlier tests. Body mechanics are affected by both the physical 
parameters of the lifting task and the behavior of the evaluee, which is evaluated through the use of "highrisk work style" (HRWS) measurement guidelines applied during the lift. Instructions must be read verbatim or minimally paraphrased. A psychophysical performance target is provided in the instructions. The evaluee is asked to rate the perceived load before being asked whether or not he/she would be able to perform the lifting task on a "safe and dependable basis eight to twelve times per day" immediately after each task cycle. Heart rate is monitored continuously. Performance on a sub-test cannot begin if the evaluee is at or above $70 \%$ of age-based predicted maximum heart rate (PMHR). In addition, performance is immediately stopped if the evaluee achieves $85 \%$ of PMHR. A scheme to restrict loads to a proportion of ideal body weight based on gender and height is used, with a separate limit for each sub-test for each gender. Thus, there are twelve such guidelines. These guidelines are used to identify the point at which the load exceeds a generally acceptable maximum based on ergonomics research. If there is a good reason to exceed this load and if the evaluee's performance indicates that it would be safe, the test can proceed. If either of these conditions is not met, the test must be terminated. As previously described, the ELC test protocol can be terminated for many reasons including surpassing safe physiological, psychological and biomechanical limits [35]. The Maximum Acceptable Weight (MAW) is determined by the evaluator based on these guidelines and procedures. For the present study, a target load was not used; each participant was tested to his or her safe and dependable maximum acceptable weight.

\section{Materials and methods}

The EPIC Lift Capacity (ELC) test has demonstrated safety and reliability $[20,35,40]$ that led to its use in a functional capacity evaluation test battery in a multi-site study in California [41]. Subsequent studies by this research team examined the construct validity and utility of the ELC in treatment settings [25], finding that aerobic capacity and back strength made significant independent contributions to lift capacity [42]. Mooney and colleagues [43] found that the combination of self-reported physical function as assessed by the Multidimensional Task Ability Profile test, gender, and age accounted for $67 \%$ of the variance in lift capacity. The purpose of the current large scale study $(n=$ 4,443 ) is to confirm safety, reliability, and validity as well as the development of normative age and gender data.

\subsection{Test design}

A test-retest design with a one-week interval was used with healthy normal participants to study the cross-sectional effects of age and gender on lift capacity. Participants were unpaid adult volunteers recruited by word of mouth during the ELC health provider certification program, after the provider completed a seven-hour formal training experience. Subjects self-reported as healthy and subsequently were screened with a structured health history questionnaire, the Cornell Medical Index [44] and provided a physical screening to rule out cardiovascular or orthopedic contraindications to exercise. Volunteers who were found to have cardiovascular signs and symptoms, hypertension (resting heart rate $>90 \mathrm{bpm}$ or resting $\mathrm{BP}>$ $159 / 100 \mathrm{~mm} \mathrm{Hg}$ ), or musculoskeletal impairment were excluded from further study.

\subsection{Variables}

Lift capacity was measured by the ELC on a progressive-demand basis, with the maximum acceptable weight (MAW) operationally defined as the load that the evaluee is able to lift "on a safe and dependable basis, eight to twelve times per day", along with the standard decision criteria followed to determine MAW that have been published previously, as depicted in the flow chart in Fig. 1.

An important aspect of the ELC training process is the development of skills used to implement this series of decision criteria to safely determine Maximum Acceptable Weight. The formal training program and formal knowledge evaluation with a 50-item test preceded the "hands-on" testing conducted by the provider with 5 volunteers on a test-retest basis that provide the data for the present study.

\subsection{Participants}

This study employed a convenience sample of 4,443 adult volunteers (41\% male), with an age range of 15 years to 65 years and mean (SD) $30.6(8.8)$ years. The participants were unpaid volunteers who were recruited by applicants for certification in the ELC certification program. All participants were unfamiliar with the ELC protocol, without history of a musculoskeletal injury or cardiovascular contraindications. National distribution data are presented in Table 1.

Canada and the United States, Great Britain, China, Australia, and New Zealand contributed participants. 
Table 1

National distribution of subjects

\begin{tabular}{lrccccccc}
\hline & \multicolumn{7}{c}{ Nation } \\
\cline { 2 - 9 } & AUS & CAN & CHN & GB & NZ & US & Unknown & Total \\
\hline Male & 48 & 1212 & 9 & 1 & 10 & 527 & 13 & 1820 \\
Female & 92 & 1752 & 16 & 4 & 17 & 723 & 19 & 2623 \\
Count & 140 & 2964 & 25 & 5 & 27 & 1250 & 32 & 4443 \\
Percent & $3.2 \%$ & $66.7 \%$ & $0.6 \%$ & $0.1 \%$ & $0.6 \%$ & $28.1 \%$ & $0.7 \%$ & $100.0 \%$ \\
\hline
\end{tabular}

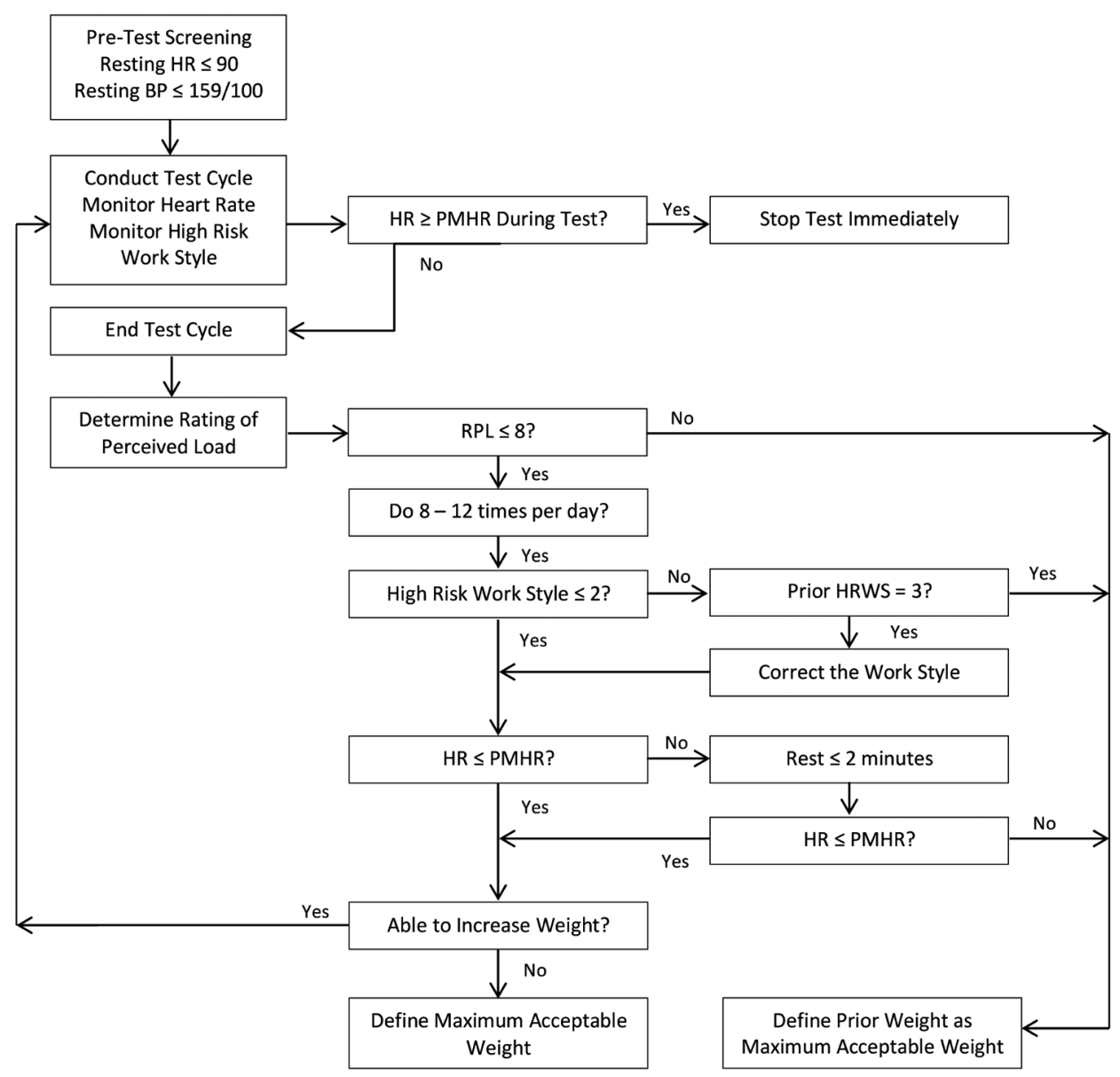

Fig. 1. Determination of maximum acceptable weight.

Participants for whom a country designation could not be made were grouped into the "unknown" category. Participants' descriptive data are presented in Table 2, with distribution of the participants by age and gender presented in Table 3.

Separate one-way analyses of variance demonstrated significant differences between males and females in terms of age $\left(F_{1,4440}=13.24, p<0.001\right)$, and height $\left(F_{1,3319}=2693.52, p<0.001\right)$ and weight $\left(F_{1,3262}=\right.$ 2405.98, $p<0.001$ ) with males being older, taller, and heavier, respectively. In this sample females and young adults predominate, with young females being especially prevalent.

\subsection{Procedures}

\subsubsection{Informed consent}

This research project was reviewed and approved by the first author's institutional review board. Prior to participation in the study, each candidate provided written informed consent using a consent process that was approved by the institutional review board. 
Table 2

Descriptive data

\begin{tabular}{llrrrcc}
\hline & & $N$ & Mean & SD & Minimum & Maximum \\
\hline Age years & Male & 1820 & 31.2 & 8.94 & 15 & 65 \\
& Female & 2622 & 30.2 & 8.74 & 15 & 64 \\
& Total & 4442 & 30.6 & 8.84 & 15 & 65 \\
Height $(\mathrm{cm})$ & Male & 1428 & 178.7 & 7.6 & 144.8 & 210.8 \\
& Female & 1893 & 165.1 & 7.2 & 132.1 & 200.7 \\
& Total & 3321 & 170.9 & 10.0 & 132.1 & 210.8 \\
Weight $(\mathrm{cm})$ & Male & 1409 & 82.2 & 12.5 & 50.0 & 159.1 \\
& Female & 1855 & 62.7 & 10.2 & 39.5 & 111.4 \\
& Total & 3264 & 71.1 & 14.8 & 39.5 & 159.1 \\
\hline
\end{tabular}

Table 3

Distribution by age and gender

\begin{tabular}{lcccccc}
\hline & \multicolumn{5}{c}{ Age group (years) } \\
\cline { 2 - 7 } & $15<30$ & $30<40$ & $40<50$ & $50 \leqslant 60$ & $60+$ yrs & Total \\
\hline Male & 987 & 526 & 208 & 83 & 16 & 1820 \\
Female & 1617 & 606 & 269 & 119 & 11 & 2622 \\
Count & 2604 & 1132 & 477 & 202 & 27 & 4442 \\
Percent & $58.6 \%$ & $25.5 \%$ & $10.7 \%$ & $4.5 \%$ & $0.6 \%$ & $100.0 \%$ \\
\hline
\end{tabular}

Table 4

ELC subtest order

\begin{tabular}{cll}
\hline Subtest & Vertical range & Frequency \\
\hline 1 & Waist to shoulder & 1 rep/cycle \\
2 & Floor to waist & 1 rep/cycle \\
3 & Floor to shoulder & 1 rep/cycle \\
4 & Waist to shoulder & 4 reps/cycle \\
5 & Floor to waist & 4 reps/cycle \\
6 & Floor to shoulder & 4 reps/cycle \\
\hline
\end{tabular}

\subsubsection{Health questionnaire}

After the informed consent process was completed, each participant completed a 45 -item medical history questionnaire that was subsequently reviewed by the evaluator. This is composed of sections from the Cornell Medical Index health questionnaire [44] that pertain to cardiovascular and musculoskeletal health and cardiac signs and symptoms. Each completed health questionnaire was reviewed by the evaluator and submitted for review by the authors with the evaluation records and informed consent documents. Affirmative responses to questions in the participant's medical history that indicated cardiovascular signs and symptoms precluded participation in the study. Completion of the requirements for inclusion in the study was confirmed by the evaluator. Self-reported height and weight were recorded, based on participants' response to questions by the evaluator.

\subsubsection{Resting heart rate and blood pressure}

After the health questionnaire was reviewed, while the participant was seated at rest, resting heart rate and blood pressure were measured. Participants were ex- cluded if they were found to have elevated resting heart rate $(>90 \mathrm{bpm})$ or hypertension $(>159 / 100 \mathrm{~mm} \mathrm{Hg})$.

\subsubsection{Normal job demands}

Self-report data from incumbent workers to measure the physical demands of work have formed the backbone of functional job analysis since the 1950's [4548], and are the basis of the Dictionary of Occupational Titles [49-51]. While questionnaires have sometimes been found to be unreliable [52], other studies have found them to be reliable and valid [53] and are generally recognized as both useful and necessary [54]. In the current study a 12-item job demands questionnaire [55] was used to identify the physical demand characteristics level of the participant's usual and customary job based on the definitions of the strength demands of work used in the Dictionary of Occupational Titles [56], as operationally defined in the Revised Handbook for Analyzing Jobs [57]. The questions are presented in Fig. 2.

The questionnaire has been used in functional capacity evaluation $[41,58]$ and has found to be a useful tool in participatory ergonomics programs [59-61]. The questionnaire was administered to each participant immediately prior to the initial ELC test session. The physical demand characteristics rating based on the 5level Dictionary of Occupational Titles system for each participant's usual and customary job was derived after the ELC test performance data had been submitted by blind review of the completed questionnaire. This review was conducted by a data analyst using a simple protocol that confirmed consistency among responses to the 12 items in the questionnaire. When consistency was demonstrated, the response to item 12 was taken as the participant's physical demand characteristics level. When consistency was not demonstrated, the questionnaire was determined to be "inconsistent" and this datum was not entered in the participant's database.

\subsubsection{Lift capacity evaluation}

The ELC was administered in the standard manner by a wide variety of healthcare professionals who were participating in the ELC certification program. In this program, each applicant is trained to use the ELC protocol in either a one-day in-person workshop or in a 7-hour self-study program with the same printed and audiovisual materials including an examiner's manual [37-39]. After training, each applicant for certification is required to pass a written exam on the test procedures and safeguards before testing 5 healthy normal volunteers on a test-retest basis. These test records 
1. How many hours per week do you usually work on this job?

2. Work postures: Fill in the hours per day that you usually work in these postures.

Sitting down (office, car, truck, etc.).

Max at 1 Time Total Hours

Standing (at a counter, at a machine, etc.).

Walking while carrying less than 20 pounds.

Walking while carrying more than 20 pounds

Use this rating scale to answer questions $3-11$ :

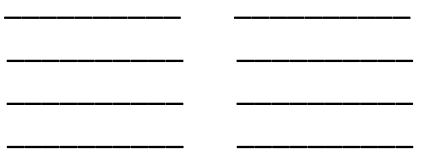

$\begin{array}{ccc}\text { ( A ) } & \text { ( B ) } & \text { (C) } \\ \begin{array}{c}\text { not at all } \\ \text { (never) }\end{array} & \text { rarely } & \text { occasionally } \\ \text { (less than } 1 / 10 & \text { (less than } 1 / 3 \\ \text { of the time) } & \text { of the time) }\end{array}$
( A )
not at all
of the time)
(less than $1 / 3$
( $E$ )
frequently
constantly
$(1 / 3$ to $2 / 3 \quad$ (more than $2 / 3$
of the time)
of the time)

3. How often do you have to kneel or crawl in your work?.

4. How often do you have to lie down (for example, as an auto mechanic) in your work?

5. How often do you have to squat or bend or twist at the hips in your work?......

6. How often do you do work which caused vibrations to your whole body? .

7. How often do you have to operate a foot pedal?....

8. On this job, how often do you lift:

10 to 20 pounds:

20 to 50 pounds:

50 to 100 pounds:

More than 100 pounds:

9. On this job, how often do you carry:

10 to 20 pounds:

20 to 50 pounds:

50 to 100 pounds:

More than 100 pounds:

10. How often do you jump from one level to another?

11. About how often per day do you climb steps on this job?

12 Five ratings of physical demands are below. Please mark the one that best describes your job.

( ) Sedentary Sometimes I stand or walk, but I sit down most of the time.

( ) Light Any of the following:

- I walk or stand more than one third of the time

- I often lift up to 10 pounds

( ) Medium I often lift up to 20 pounds, or sometimes up to 50 pounds.

( ) Heavy I often lift up to 50 pounds, or sometimes up to 100 pounds.

( ) Very Heavy I often lift over 50 pounds, or sometimes over 100 pounds.

Fig. 2. Job demands questionnaire.

were submitted for review to assure adherence to standardized test procedures and understanding of the decision criteria to determine maximum acceptable weight (MAW). The data from the certification program were used in the current study. In the standard ELC administration, each participant's heart rate is continuously monitored and documented. Maximum acceptable weight is measured in six separate subtests for each participant, as depicted in Table 4. Following the standard ELC protocol, standing rest breaks of two minutes were provided between subtests. The measure of lift capacity was the Maximum Acceptable Weight (MAW) for each of the six subtests. Follow-up testing was conducted mean (SD) 5.8 (5.9) days after the initial testing (range 1 to 70 days), using the same testing protocol. The MAW for each subtest in the initial test 
Table 5

Subjects' maximum acceptable weight (kilograms) across all subtests

\begin{tabular}{|c|c|c|c|c|c|c|c|c|}
\hline \multirow[b]{3}{*}{ Subtest } & \multicolumn{4}{|c|}{ Male } & \multicolumn{4}{|c|}{ Female } \\
\hline & \multicolumn{2}{|c|}{ Test } & \multicolumn{2}{|c|}{ Retest } & \multicolumn{2}{|c|}{ Test } & \multicolumn{2}{|c|}{ Retest } \\
\hline & Mean & SD & Mean & $\mathrm{SD}$ & Mean & $\mathrm{SD}$ & Mean & SD \\
\hline 1 & 33.74 & 7.74 & 33.88 & 7.82 & 19.54 & 5.79 & 19.89 & 6.13 \\
\hline 2 & 38.50 & 8.26 & 38.33 & 8.29 & 24.70 & 7.18 & 24.56 & 7.36 \\
\hline 3 & 34.24 & 7.80 & 34.34 & 7.77 & 19.29 & 5.82 & 19.67 & 5.80 \\
\hline 4 & 27.74 & 6.83 & 28.20 & 6.97 & 15.82 & 5.07 & 16.34 & 5.05 \\
\hline 5 & 31.26 & 7.93 & 31.77 & 7.69 & 19.44 & 6.43 & 20.03 & 6.94 \\
\hline 6 & 26.39 & 7.28 & 27.07 & 6.91 & 14.86 & 5.20 & 15.51 & 5.09 \\
\hline
\end{tabular}

Table 6

Subjects' response to retest

\begin{tabular}{|c|c|c|c|c|c|c|c|c|c|c|c|c|}
\hline \multirow[b]{2}{*}{ Source } & \multicolumn{2}{|c|}{ Subtest \#1 } & \multicolumn{2}{|c|}{ Subtest \#2 } & \multicolumn{2}{|c|}{ Subtest \#3 } & \multicolumn{2}{|c|}{ Subtest \#4 } & \multicolumn{2}{|c|}{ Subtest \#5 } & \multicolumn{2}{|c|}{ Subtest \#6 } \\
\hline & $F$ & Sig. & $F$ & Sig. & $F$ & Sig. & $F$ & Sig. & $F$ & Sig. & $\bar{F}$ & Sig. \\
\hline Retest & 5.28 & 0.022 & 0.68 & 0.408 & 11.79 & 0.001 & 10.51 & 0.001 & 3.46 & 0.063 & 0.94 & 0.331 \\
\hline Retest $\times$ Age & 1.30 & 0.254 & 2.55 & 0.110 & 4.95 & 0.026 & 0.50 & 0.481 & 0.07 & 0.785 & 5.07 & 0.024 \\
\hline Retest $\times$ Sex & 3.04 & 0.082 & 0.02 & 0.895 & 6.59 & 0.010 & 0.27 & 0.605 & 0.34 & 0.560 & 0.00 & 0.962 \\
\hline
\end{tabular}

Table 7

Test-retest $\operatorname{ICC}_{(2,1)}$ maximum acceptable weight; $95 \%$ confidence intervals

\begin{tabular}{cccc}
\hline Subtest & ICC & Lower bound & Upper bound \\
\hline 1 & 0.925 & 0.920 & 0.929 \\
2 & 0.926 & 0.921 & 0.930 \\
3 & 0.938 & 0.934 & 0.941 \\
4 & 0.910 & 0.991 & 0.915 \\
5 & 0.865 & 0.857 & 0.872 \\
6 & 0.890 & 0.884 & 0.896 \\
\hline
\end{tabular}

administration was used as the datum for the normative data analyses in this study.

\subsubsection{Symptom follow-up}

Immediately prior to follow-up testing, each participant completed a questionnaire that screened for any symptoms or injuries that were encountered during the initial occasion of testing. If symptoms were reported, the questionnaire was reviewed by the evaluator before proceeding to the retest.

\section{Data analysis}

Analysis of variance and repeated measures analysis of variance were used to examine group-wise differences among participants. The Intraclass Correlation $\operatorname{ICC}_{(2,1)}$ procedure was used to examine reliability and stability of the data. Frequency analyses for each subtest within each gender and age group were used to determine normative data. Significance level was set at $p=0.01$. Data were maintained in Excel $^{\mathrm{TM}}$ spreadsheets and analyzed with SPSS Statistics ${ }^{\mathrm{TM}}$ version 19. Guidelines regarding interpretation of correlation co- efficients follow Portney and Watkins [62], in which coefficients below 0.50 represent poor reliability, coefficients from 0.50 to 0.75 indicate moderate reliability, and values above 0.75 indicate good reliability.

\section{Results}

In this phase of the ELC Certification program, 912 evaluators contributed 4,443 complete test-retest data sets. While most of the evaluators were occupational therapists, physical therapists, and kinesiotherapists, other healthcare professionals such as medical physicians, chiropractors, athletic trainers, registered nurses, vocational evaluators, and rehabilitation psychologists were also involved in the training and certification program.

\subsection{Safety}

Overall, the ELC test was administered to 4,443 participants. No serious adverse events were reported. Side effects were rare, typically minor, and temporary. Of the 4,443 participants who began the initial testing, 4,440 (99.9\%) were able to complete the first three subtests in the battery and 4,375 (98.5\%) were able to complete all six subtests. Of the 68 participants who did not complete the full battery, 39 completed all but Subtest \#6. Typical reasons for not completing all six subtests were that the participant's heart rate did not recover sufficiently within the time allowed to permit continuing or that maximum acceptable weight on either of the frequent subtests was too low to al- 
Table 8

Lift capacity (kilograms) by gender and age group

\begin{tabular}{|c|c|c|c|c|c|c|c|c|c|c|c|c|c|}
\hline & \multirow[b]{2}{*}{ Age (years) } & \multicolumn{2}{|c|}{ Sub-test 1} & \multicolumn{2}{|c|}{ Sub-test 2} & \multicolumn{2}{|c|}{ Sub-test 3} & \multicolumn{2}{|c|}{ Sub-test 4} & \multicolumn{2}{|c|}{ Sub-test 5} & \multicolumn{2}{|c|}{ Sub-test 6} \\
\hline & & Mean & $\mathrm{SD}$ & Mean & $\mathrm{SD}$ & Mean & SD & Mean & SD & Mean & $\mathrm{SD}$ & Mean & SD \\
\hline \multirow{5}{*}{ Male } & $15<30$ & 34.4 & 7.4 & 39.1 & 7.9 & 35.0 & 7.6 & 28.3 & 6.6 & 32.0 & 7.5 & 27.0 & 7.3 \\
\hline & $30<40$ & 33.7 & 7.7 & 38.5 & 8.2 & 34.1 & 7.6 & 27.6 & 6.8 & 31.1 & 8.0 & 26.3 & 7.3 \\
\hline & $40<50$ & 33.4 & 7.9 & 38.1 & 8.5 & 33.5 & 7.8 & 27.6 & 7.2 & 30.8 & 8.5 & 25.3 & 7.4 \\
\hline & $50<60$ & 28.9 & 8.5 & 33.5 & 9.2 & 29.5 & 8.8 & 23.7 & 7.4 & 25.7 & 8.8 & 20.5 & 8.8 \\
\hline & $60+\mathrm{yrs}$ & 25.1 & 6.9 & 31.5 & 10.3 & 26.6 & 8.3 & 20.2 & 7.2 & 21.4 & 9.7 & 17.9 & 7.0 \\
\hline \multirow[t]{5}{*}{ Female } & $15<30$ & 19.8 & 5.7 & 25.3 & 7.0 & 19.6 & 5.7 & 15.9 & 5.0 & 19.9 & 6.3 & 15.0 & 5.1 \\
\hline & $30<40$ & 19.9 & 5.7 & 25.1 & 7.1 & 19.6 & 5.7 & 16.3 & 5.2 & 19.8 & 6.4 & 15.1 & 5.6 \\
\hline & $40<50$ & 18.3 & 5.7 & 22.3 & 7.3 & 17.6 & 5.9 & 14.7 & 5.2 & 17.4 & 6.5 & 13.3 & 5.5 \\
\hline & $50<60$ & 16.9 & 6.0 & 20.6 & 7.1 & 16.5 & 6.3 & 13.2 & 5.7 & 15.6 & 6.7 & 12.3 & 6.4 \\
\hline & $60+y r s$ & 17.4 & 5.2 & 20.0 & 7.5 & 16.5 & 5.5 & 13.2 & 4.6 & 15.3 & 5.6 & 12.2 & 4.5 \\
\hline
\end{tabular}

Table 9

Lift capacity adequacy: Comparing lift capacity with job demands

\begin{tabular}{|c|c|c|c|c|c|c|c|c|}
\hline & \multirow[b]{2}{*}{ PDC } & \multicolumn{5}{|c|}{ Job demand } & \multirow[b]{2}{*}{ Total } & \multirow[b]{2}{*}{$\%$ Inadequate } \\
\hline & & Sedentary & Light & Medium & Heavy & Very heavy & & \\
\hline \multirow[t]{6}{*}{ Lift capacity } & Sedentary & 11 & 22 & 6 & 1 & 0 & 40 & $72.50 \%$ \\
\hline & Light & 39 & 22 & 11 & 0 & 0 & 72 & $15.28 \%$ \\
\hline & Medium & 255 & 239 & 75 & 4 & 2 & 575 & $1.04 \%$ \\
\hline & Heavy & 595 & 820 & 294 & 26 & 8 & 1743 & $0.46 \%$ \\
\hline & Very heavy & 242 & 387 & 206 & 66 & 19 & 920 & $0.00 \%$ \\
\hline & Total & 1133 & 1471 & 586 & 96 & 29 & 3350 & $1.40 \%$ \\
\hline
\end{tabular}

Table 10

Lift capacity across job demand categories

\begin{tabular}{|c|c|c|c|c|c|c|}
\hline & PDC & $N$ & Mean & $\mathrm{SD}$ & Minimum & Maximum \\
\hline \multirow[t]{6}{*}{ Job demand } & Sedentary & 1142 & 17.6 & 8.0 & 0 & 45.5 \\
\hline & Light & 1490 & 19.0 & 8.0 & 0 & 50.0 \\
\hline & Medium & 592 & 21.2 & 8.8 & 0 & 52.3 \\
\hline & Heavy & 97 & 26.5 & 7.8 & 0 & 45.5 \\
\hline & Very heavy & 29 & 27.9 & 8.8 & 9.1 & 40.9 \\
\hline & Total & 3350 & 19.2 & 8.4 & 0 & 52.3 \\
\hline
\end{tabular}

low Subtest \#6 to be undertaken. Another 14 participants reported symptoms of pain that caused the evaluator to stop the test before its conclusion. All of these participants returned for retest. On return, each completed the symptom follow-up questionnaire and none reported that the pain had indicated an injury; all pain had resolved without medical intervention other than over-the-counter non-steroidal anti-inflammatory medication or acetaminophen.

\subsection{ELC Test performance}

A series of one-way analysis of variance based on gender demonstrated a significant difference in terms of maximum acceptable weight for all six subtests in the ELC battery on both the test and retest. Men consistently demonstrated greater capacity than women on all tests on both testing occasions (all $p<0.0001$ ) (Table 5).
Repeated measures analysis of variance of each subtest based on gender with age as a covariate confirmed significant effects for age and gender (all $p<0.001$ ). There is also a significant effect for retest on Subtest \#3 and Subtest \#4, with a significant interaction between retest and gender on Subtest \#3 (Table 6).

\subsection{Reliability}

The results of separate analyses using the $\operatorname{ICC}_{(2,1)}$ Shrout and Fleiss [64] convention are presented in Table 7 .

\subsection{Validity}

Performance in the ELC subtests was studied in terms of both age and gender, the results of which are presented in Table 8 .

Main effects for both age group and gender are found for each of the subtests, (all $p<0.001$ ). Across all subtests, men were stronger than women, and younger subjects were stronger than older subjects.

An important use of the ELC data is to determine the adequacy of a person's lift capacity for his or her job's demands. In the current research, this was addressed by comparing the participant's responses to the job demands questionnaire administered prior to the initial ELC test with his or her lift capacity as measured by 
Table 11

Lift capacity normative data (kilograms)

\begin{tabular}{|c|c|c|c|c|c|c|c|c|c|}
\hline & \multirow[b]{2}{*}{ Percentile } & \multicolumn{4}{|c|}{ Male } & \multicolumn{4}{|c|}{ Female } \\
\hline & & $15<30$ yrs & $30<40$ yrs & $40<50$ yrs & $50<60$ yrs & $15<30$ yrs & $30<40$ yrs & $40<50$ yrs & $50<60$ yrs \\
\hline \multirow[t]{5}{*}{ Subtest 1} & $90^{\text {th }}$ & 45 & 45 & 45 & 41 & 27 & 27 & 27 & 23 \\
\hline & $75^{\text {th }}$ & 41 & 41 & 41 & 36 & 23 & 23 & 23 & 18 \\
\hline & $50^{\text {th }}$ & 36 & 36 & 36 & 27 & 18 & 18 & 18 & 18 \\
\hline & $25^{\text {th }}$ & 27 & 27 & 27 & 23 & 14 & 16 & 14 & 14 \\
\hline & $10^{\text {th }}$ & 23 & 23 & 23 & 18 & 14 & 14 & 9 & 9 \\
\hline \multirow[t]{5}{*}{ Subtest 2} & $90^{\text {th }}$ & 45 & 45 & 45 & 45 & 36 & 32 & 32 & 32 \\
\hline & $75^{\text {th }}$ & 45 & 45 & 45 & 41 & 32 & 32 & 27 & 27 \\
\hline & $50^{\text {th }}$ & 41 & 41 & 41 & 36 & 27 & 27 & 23 & 18 \\
\hline & $25^{\text {th }}$ & 36 & 32 & 32 & 27 & 18 & 18 & 18 & 14 \\
\hline & $10^{\text {th }}$ & 27 & 27 & 27 & 23 & 18 & 14 & 14 & 9 \\
\hline \multirow[t]{5}{*}{ Subtest 3} & $90^{\text {th }}$ & 43 & 43 & 43 & 41 & 27 & 27 & 25 & 25 \\
\hline & $75^{\text {th }}$ & 41 & 39 & 39 & 36 & 23 & 23 & 20 & 20 \\
\hline & $50^{\text {th }}$ & 36 & 34 & 34 & 30 & 18 & 20 & 18 & 16 \\
\hline & $25^{\text {th }}$ & 32 & 30 & 27 & 23 & 16 & 14 & 14 & 14 \\
\hline & $10^{\text {th }}$ & 25 & 25 & 23 & 18 & 14 & 14 & 9 & 9 \\
\hline \multirow[t]{5}{*}{ Subtest 4} & $90^{\text {th }}$ & 36 & 34 & 34 & 32 & 23 & 23 & 23 & 20 \\
\hline & $75^{\text {th }}$ & 32 & 32 & 32 & 27 & 18 & 20 & 18 & 16 \\
\hline & $50^{\text {th }}$ & 30 & 27 & 27 & 25 & 16 & 16 & 14 & 14 \\
\hline & $25^{\text {th }}$ & 23 & 23 & 23 & 18 & 14 & 14 & 11 & 9 \\
\hline & $10^{\text {th }}$ & 20 & 18 & 18 & 14 & 9 & 9 & 9 & 7 \\
\hline \multirow[t]{5}{*}{ Subtest 5} & $90^{\text {th }}$ & 41 & 41 & 41 & 36 & 27 & 27 & 27 & 25 \\
\hline & $75^{\text {th }}$ & 36 & 36 & 36 & 32 & 25 & 25 & 23 & 18 \\
\hline & $50^{\text {th }}$ & 34 & 32 & 32 & 25 & 20 & 20 & 18 & 16 \\
\hline & $25^{\text {th }}$ & 27 & 27 & 25 & 18 & 16 & 16 & 14 & 9 \\
\hline & $10^{\text {th }}$ & 23 & 20 & 18 & 16 & 11 & 11 & 9 & 7 \\
\hline \multirow[t]{5}{*}{ Subtest 6} & $90^{\text {th }}$ & 34 & 34 & 34 & 32 & 23 & 23 & 20 & 20 \\
\hline & $75^{\text {th }}$ & 32 & 32 & 32 & 27 & 18 & 18 & 16 & 16 \\
\hline & $50^{\text {th }}$ & 27 & 27 & 27 & 20 & 16 & 16 & 14 & 11 \\
\hline & $25^{\text {th }}$ & 23 & 23 & 20 & 16 & 11 & 11 & 9 & 9 \\
\hline & $10^{\text {th }}$ & 18 & 16 & 16 & 9 & 9 & 9 & 7 & 5 \\
\hline
\end{tabular}

the initial ELC Subtest \#6. The five-level Physical Demand Characteristics of work system [57] was used to classify both (Table 9).

In Table 9, the shaded cells indicate mismatches in which the participant's lift capacity was inadequate for the job's demands. In this set of 3,350 participants, $1.4 \%$ of the participants were found to have inadequate lift capacity. Another way to look at these data is on a group-wise basis with analysis of variance, considering participants' lift capacity grouped by the PDC level of their jobs (Table 10).

A one-way analysis of variance demonstrated significant differences among the job categories in terms of lift capacity as demonstrated in Subtest \#6 $\left(F_{4,3310}=\right.$ 50.43, $p<0.001)$. As job demands increase, the average lift capacity of the participant in the job increases, reflecting the combined effects of job participation in developing and maintaining lift capacity and the selection of stronger persons into the more demanding jobs.

Normative data were based on frequency analyses and are presented as percentile rankings of MAW for each subtest across each gender and each age group in Table 11.

\section{Discussion}

The ELC is used within the context of professional guidelines as well as numerous state and federal laws. Guidelines for testing have been published by the American Psychological Association [36], the American Physical Therapy Association [64], and the American Academy of Physical Medicine and Rehabilitation [65]. There is agreement among these professional entities that use of a test must be undertaken within the context of these standards:

Safety - Given the known characteristics of the evaluee, when used properly the test should not be expected to lead to injury. This study demonstrated that the ELCcan be administered safely by trained professionals in several health care disciplines. 
Reliability - Reliability is the dependability of the test over time and between evaluators. This research demonstrates good reliability of the ELC on a test-retest basis for all ELC subtests.

Validity - Validity is the extent to which the measure of performance is dependably related to a criterion $[36,64,65]$. The use of the ELC to determine adequacy for the evaluee's self-reported physical demands of work, to reflect age and gender differences, and to be consistent with ergonomic demands posed by vertical lifting range and lift frequency has been demonstrated in this study.

Normative data for hand strength and physical fitness are useful in health care and medical science. In exercise science, there has been an interest in using standardized reliable measures to collect age-relevant normative performance data [66]. Without standard measures, it was not possible to communicate energy costs of aerobic exercise in common language. Normative data were developed through standardized testing protocols, which when repeated verified their reliability and validity [66,67]. The American College of Sports Medicine used these data to recommend different levels of exercise to achieve and maintain health [68]. The current study's measure of lift capacity provides a useful addition to the constellation of normative data available to assess healthy adults.

The age-based differences in lift capacity in the ELC normative data are consistent with those reported in the classic paper by Fisher and Birren [69] and many others, indicating that strength appears to peak in the late 20's and decreases slowly with maturity. This also compares well with several studies from the field of ergonomics [70-74], and helps to emphasize the importance of attending to age-wise and gender-based differences in lift capacity.

Comparison of the current findings to the work of other researchers who have studied the maximum acceptable weight of lift to develop safe work standards is difficult because these studies [75-80] have not included the effects of age and body mass and are usually based on small sample sizes, with subjects who are young or in early middle age and of average weight. Such studies do not allow an opportunity to take a look at age effects, the combination of age and gender effects, or the effect of body mass, all of which have been demonstrated to be important predictors of lift capacity [20].

As the workforce ages, becomes gender-balanced, and experiences increased body mass per worker, these factors must be included in models that are used to design safe jobs [81,82]. Normative data of all types are useful in industrial settings to design jobs that are likely to be safely performed by people of all ages and both genders. Data provided in this study may be useful in that regard.

\section{Summary}

This study of lift capacity as assessed by the ELC of healthy normal volunteers demonstrated safety in use of the test across a wide range of health care and rehabilitation professionals who have participated in formal training. Analysis of the data with regard to reliability on a test-retest basis across each of the six subtests in the battery demonstrated good reliability for both lift capacity measures. Validity for the adequacy of lift capacity in terms of the self-reported physical demands of the participant's usual and customary job was established. The effects of age and gender that are reported in the scientific literature also were found in this study. Given these effects, normative data from the test session are presented separately across gender for each age category and each sub-test. Retest normative data were not presented because most applications of ELC test data will be with persons who are naïve to the test.

This is the first large-scale study of lift capacity to include simultaneous examination of the test-retest reliability and validity of test performance, which is an obvious strength; the normative data can be confirmed as stable over time and related to self-reported physical demands of the usual and customary job.

In terms of limitations, this study used a convenience sample, which leads to restriction in use of the data to the age and gender categories that were wellrepresented. Additionally, most of the participants in the study were from North America, which presents another restriction on the use of this study's normative data.

\section{Disclosure}

Dr. Matheson has a proprietary interest in the company that developed and licenses the EPIC Lift Capacity test, and receives financial benefits from its use. Neither Dr. Verna nor Dr. Dreisinger, nor Dr. Mayer nor Mr. Leggett have a proprietary interest in the EPIC Lift Capacity test, or receive financial benefits from its use. 


\section{Acknowledgments}

The authors wish to acknowledge the contributions to data collection by Karen Markley, Lisa Markley, Sherry McCowan and Kristen Del Real of EpicRehab, and Monique Lopez of the Vert Mooney Research Foundation.

\section{References}

[1] Innes E, Straker L. Reliability of work-related assessments. Work. 1999; 13: 107-124.

[2] Innes E, Straker L. Validity of work-related assessments. Work. 1999; 13: 125-152.

[3] Innes E, Straker L. Attributes of excellence in work related assessments. Work. 2003; 20: 63-76.

[4] Innes E. Reliability and validity of functional capacity evaluations: An update. International Journal of Disability Management Research. 2006; 1(1): 135-148.

[5] Innes E, Straker L. A clinician's guide to work-related assessments. Work. 1998; 11: 183-189.

[6] Gross DP, Battie MC, Cassidy JD. The prognostic value of functional capacity evaluation in patients with chronic low back pain: part 1: timely return to work. Spine. 2004; 29(8): 914-949.

[7] Matheson LN, Isernhagen SJ, Hart DL. Relationships among lifting ability, grip force, and return to work. Physical Therapy. 2002 Mar; 82(3): 249-256.

[8] Gross DP, Battie MC. The prognostic value of functional capacity evaluation in patients with chronic low back pain: part 2: sustained recovery. Spine. 2004; 29(8): 920-924.

[9] Gross DP, Battie MC. Functional capacity evaluation performance does not predict sustained return to work in claimants with chronic back pain. Journal of Occupational Rehabilitation. 2005 Sep; 15(3): 285-294.

[10] Matheson L, Carlton R, Niemeyer L. Grip strength in a disabled sample: Reliability and normative standards. Industrial Rehabilitation Quarterly. 1988; 1(3): 17-23.

[11] Mathiowetz V, Kashman N, Volland G, Weber K, Dowe M, Rogers S. Grip and pinch strength: Normative data for adults. Archives of Physical Medicine and Rehabilitation. 1985; 6: 69-74.

[12] Schmidt R, Toews J. Grip strength as measured by the Jamar dynamometer. Archives of Physical Medicine and Rehabilitation. 1970; 51: 321-327.

[13] Åstrand PO. Quantification of exercise capability and evaluation of physical capacity in man. Prog Cardiovasc Dis. 1976 Jul-Aug; 19(1): 51-67.

[14] Shvartz E, Reibold R. Aerobic fitness norms for males and females aged 6-75: A review. Aviation, Space and Environmental Medicine. 1990; 61: 3-11.

[15] van der Velde G, Mierau D. The effect of exercise on percentile rank aerobic capacity, pain, and self-rated disability in patients with chronic low-back pain: A retrospective chart review. Archives of Physical Medicine and Rehabilitation. 2000; 81(11): 1457-1463.

[16] Lobo A, Carvalho J, Santos P. Comparison of functional fitness in elderlies with reference values by Rikli and Jones and after one-year of health intervention programs. J Sports Med Phys Fitness. 2011 Mar; 51(1): 111-120.
[17] Birren J, Schaie K, editors. Handbook of the Psychology of Aging. New York: Van Nostrand Reinhold Co; 1977.

[18] Birren J. The Psychology of Aging. New Jersey: PrenticeHall; 1964.

[19] Matheson L. Evaluation of lifting and lowering capacity. Vocational Evaluation and Work Adjustment Bulletin. 1986; 19(4): 107-111.

[20] Matheson L. Relationships among age, body weight, resting heart rate, and performance in a new test of lift capacity. Journal of Occupational Rehabilitation. 1996; 6: 225-237.

[21] Mayer T, Barnes D, Kishino N, Nichols G, Gatchel R, Mayer $\mathrm{H}$, et al. Progressive isoinertial lifting evaluation. I. a standardized protocol and normative database. Spine. 1988; 13: 993-997.

[22] NIOSH. Work practices guide for manual lifting. Cincinnati, Ohio: Division of Biomedical and Behavioral Science, NIOSH; 1981.

[23] Kumar S, Mital A. Margin of safety for the human back: A probable consensus based on published studies. Ergonomics. 1992; 35(7-8): 769-781.

[24] Matheson L, Ogden L, Violette K, Schultz K. Work hardening: Occupational therapy in industrial rehabilitation. American Journal of Occupational Therapy. 1985; 39(5): 314-321.

[25] Leggett S, Mooney V, Matheson LN, Nelson B, Dreisinger T, Van Zytveld J, et al. Restorative exercise for clinical low back pain: A prospective two-center study with 1-year followup. Spine. 1999; 24(9): 889-898.

[26] Mayer T, GatchelRJ, Kishino N, Kelley J, Capra P, Mayer H, et al. Objective assessment of spine function following industrial injury: A prospective study with comparison group and one-year follow-up. Spine. 1985; 10(6): 482-493.

[27] Mayer T, Gatchel R, Mayer H, Kishino N, Keeley J, Mooney $\mathrm{V}$. A prospective two-year study of functional restoration in industrial low back injury: An objective assessment procedure. JAMA. 1987; 258(13): 1763-1767.

[28] Isernhagen SJ. Physical therapy and occupational rehabilitation. Journal of Occupational Rehabilitation. 1991; 1(1): 7182.

[29] EEOC. Uniform guidelines on employee selection procedures (1978). Federal Register. 1993 (July 1): 212-239.

[30] Kuijer W, Dijkstra PU, Brouwer S, Reneman MF, Groothoff JW, Geertzen JH. Safe lifting in patients with chronic low back pain: comparing FCE lifting task and Niosh lifting guideline. J Occup Rehabil. 2006 Dec; 16(4): 579-589.

[31] Gibson L, Strong J. Safety issues in functional capacity evaluation: Findings from a trial of a new approach for evaluating clients with chronic back pain. Journal of Occupational Rehabilitation. 2005; 15(2): 237-251. PubMed PMID: 2009196331.

[32] Mayer T, Gatchel R, Mooney V. Safety of the dynamic progressive isointertial lifting. Spine. 1990; 15(9): 985-986.

[33] Reneman MF, Dijkstra PU, Westmaas M, Goeken LNH. Testretest reliability of lifting and carrying in a 2-day functional capacity evaluation. Journal of Occupational Rehabilitation. 2002 Dec; 12(4): 269-275.

[34] Gouttebarge V, Wind H, Kuijer PP, Sluiter JK, Frings-Dresen MH. Reliability and agreement of 5 Ergo-Kit functional capacity evaluation lifting tests in subjects with low back pain. Arch Phys Med Rehabil. 2006 Oct; 87(10): 1365-1370.

[35] Matheson L, Mooney V, Grant J, Affleck M, Hall H, Melles $\mathrm{T}$, et al. A test to measure lift capacity of physically impaired adults. Part 1 Development and reliability testing. Spine. 1995; 20: 2119-2129.

[36] American Psychological Association, American Educational 
Research Association, National Council on Measurement in Education. Standards for educational and psychological testing. Washington, DC: Author; 1999. 194 p.

[37] Matheson L. EPIC Lift Capacity Test Examiner's Manual. Fort Bragg, CA: Work Evaluation Systems Technology; 1994.

[38] Matheson L. EPIC Lift Capacity Examiner's Manual. Wildwood, MO: Employment Potential Improvement Corporation; 2003. 103 p.

[39] Matheson L. EPIC Lift Capacity Examiner's Manual. St. Charles, MO: EpicRehab; 2012. 110 p.

[40] Matheson L, Mooney V, Holmes D, Leggett M, Grant J, Negri $\mathrm{S}$, et al. A test to measure lift capacity of physically impaired adults. Part 2 Reactivity in a patient sample. Spine. 1995; 20: 2130-2134.

[41] Matheson L, Mooney V, Grant J, Leggett S, Kenny K. Standardized evaluation of work capacity. Journal of Back and Musculoskeletal Rehabilitation. 1996; 6: 249-264.

[42] Matheson L, Leggett S, Mooney V, Schneider K, Mayer J. Contributions of aerobic fitness and back strength to lift capacity. Spine. 2002; 27(11): 1208-1212.

[43] Mooney V, Matheson LN, Verna J, Leggett S, Dreisinger TE, Mayer JM. Performance-integrated self-report measurement of physical ability. Spine J. 2010 May; 10(5): 433-440.

[44] Brodman K, Erdmann A, Wolff H. Cornell Medical Index Health Questionnaire Manual. New York, NY: Cornell University Medical College; 1949. 15 p.

[45] Fleishman E, Quaintance M. Taxonomies of Human Performance. 2nd ed. Bethesda, MD: Management Research Institute, Inc; $1995.514 \mathrm{p}$.

[46] Fleishman E. Evaluating physical abilities required by jobs. The Personnel Administrator. 1979 (June): 82-87.

[47] Fleishman E, Hogan J. A taxonomic method for assessing the physical requirements of jobs: the physical abilities analysis approach (Technical Report). Washington, DC: Advanced Research Resources Organization; 1978. 44 p.

[48] Fleishman E. Relating individual differences to the dimensions of human tasks. Ergonomics. 1978; 21(12): 1007-1019.

[49] Fine S, Cronshaw S. Functional job analysis: A foundation for human resources management. Mahwah, NJ: Erlbaum; 1999.

[50] Fine S, Getkate M. Benchmark Tasks for Job Analysis: A Guide for Functional Job Analysis. 1st ed. Fleishman E, editor. Mahwah, New Jersey: Lawrence Erlbaum Associates; 1995.

[51] Fine S. What is occupational information? Personnel and Guidance Journal. 1955; 33(9): 453-468.

[52] Wiktorin C, Karlqvist L, Winkel J. Validity of self-reported exposures to work postures and manual materials handling. Stockholm MUSIC I Study Group. Scand J Work Environ Health. 1993 Jun; 19(3): 208-214.

[53] Pope DP, Silman AJ, Cherry NM, Pritchard C, Macfarlane GJ. Validity of a self-completed questionnaire measuring the physical demands of work. Scand J Work Environ Health. 1998 Oct; 24(5): 376-385.

[54] Stock SR, Fernandes R, Delisle A, Vezina N. Reproducibility and validity of workers' self-reports of physical work demands. Scand J Work Environ Health. 2005 Dec; 31(6): 409437.

[55] Evanoff B. Job Demands Questionnaire. St. Louis, MO: Washington University School of Medicine; 1993.

[56] U.S. Department of Labor. Dictionary of Occupational Titles. 4th ed. Washington, DC: United States Government Printing Office; 1991.

[57] U.S. Department of Labor. The revised handbook for analyzing jobs. Washington, DC: Author; 1991.
[58] Mooney V, Matheson L. California Functional Capacity Protocol (Cal-FCP) Examiner's Manual. San Diego, CA: Orthomed Foundation; 1994.

[59] Rhomberg S, Wolf L, Evanoff B. An integrated program for the prevention and management of musculoskeletal work injuries. WORK. 1995; 5(2): 115-122.

[60] Bohr P, Evanoff B, Wolf L. Implementing participatory ergonomics teams among health care workers. American Journal of Industrial Medicine. 1997; 32: 190-196.

[61] Evanoff BA, Bohr PC, Wolf LD. Effects of a participatory ergonomics team among hospital orderlies. American Journal of Industrial Medicine. 1999 Apr; 35(4): 358-365.

[62] Portney L, Watkins M. Foundations of Clinical Research: Applications to Practice. 2nd ed. Upper Saddle River, New Jersey: Prentice-Hall, Inc; 2000. 742 p.

[63] Shrout P, Fleiss J. Intraclass correlation: Uses in assessing rater reliability. Psychological Bulletin. 1979; 86: 420.

[64] Rothstein J, Campbell S, Echternach J, Jette A, Knecht H, Rose S. Standards for tests and measurements in physical therapy practice. In: Rothstein J, Echternach J, editors. Primer on Measurement. reprinted from Physical Therapy (71), 1991, 589-621. Alexandria, VA: American Physical Therapy Association; 1993. p. 3-47.

[65] Johnston M, Keith R, Hinderer S. Measurement standards for interdisciplinary medical rehabilitation. Archives of Physical Medicine Rehabilitation. 1992; 73: S3-S23.

[66] Åstrand I, Åstrand P, Hallbäck, Kilbom Å. Reduction in maximal oxygen uptake with age. J Appl Physiol. 1973; 35(5): 649-654.

[67] Bruce R. Exercise testing for ventricular function. New England Journal of Medicine. 1977; 296: 671-675.

[68] American College of Sports Medicine. The recommended quantity and quality of exercise for developing and maintaining cardiorespiratory and muscular fitness, and flexibility in healthy adults. Med Sci Sports Exerc. 1998 Jun; 30(6): 975991.

[69] Fisher MB, Birren JE. Age and strength. J Appl Psychol. 1947; 31: 490-497.

[70] Vitasalo J, Era P, Leskinen A, Heikkenen E. Muscular strength files and anthropology in random samples of men aged 3135, 51-55, and 71-75 years. Ergonomics. 1985 1985; 28(11): $1563-1574$.

[71] Garg A. Ergonomics and the older worker: An overview. Developmental Aging Research. 1991; 17(S3): 143-155.

[72] Garg A, Saxena U. Effects of lifting frequency and technique on physical fatigue with special reference to psychophysical methodology and metabolic rate. Am Ind Hyg Assoc J. 1979; 49: 894-903.

[73] Garg A, Mital A, Asfour S. A comparison of isometric strength and dynamic lifting capability. Ergonomics. 1980; 23(1): 13-27.

[74] Freivalds A, Chaffin D, Garg A, Lee K. A dynamic biomechanical evaluation of lifting maximum acceptable loads. J Biomechanics. 1984; 17(4): 251-262.

[75] Snook S, Irvine C. Maximum acceptable weight of lift. Am Ind Hyg Assoc J. 1967; 28: 322-329.

[76] Snook S, Irvine C, Bass S. Maximum weights and work loads acceptable to male industrial workers. Am Ind Hyg Assoc J. 1970: 579-586

[77] Snook S, Ciriello V. Maximum weights and work loads acceptable to female workers. J Occup Med. 1974; 16(8): 527534.

[78] Snook S. The design of manual handling tasks. Ergonomics. 1978; 21(12): 963-985. 
[79] Ciriello V, Snook S. A study of size, distance, height, and frequency effects on manual handling tasks. Human Factors. 1983; 25(5): 473-483.

[80] Snook SH, Ciriello VM. The design of manual handling tasks: revised tables of maximum acceptable weights and forces. Ergonomics. $1991 \mathrm{Sep} ; 34(9)$ : 1197-1213.
[81] Ciriello VM, Dempsey PG, Maikala RV, O'Brien NV. Secular changes in psychophysically determined maximum acceptable weights and forces over 20 years for male industrial workers. Ergonomics. 2008 May; 51(5): 593-601.

[82] Snook SH. Future directions of psychophysical studies. Scand J Work Environ Health. 1999; 25(Suppl 4): 13-18. 